\title{
Rejoinder: A Study on the Population Structure of Democratic People's Republic of Korea
}

\author{
Saebom Jeon ${ }^{a}$, Seong Eun $\mathrm{Kim}^{b}$, Yousung Park ${ }^{1, c}$ \\ ${ }^{a}$ Department of Applied Statistics, Korea University, Korea \\ ${ }^{b}$ Economic Statistics Department, The Bank of Korea, Korea \\ ${ }^{c}$ Department of Statistics, Korea University, Korea
}

\section{Introduction}

First, we thank the Editor for inviting comments on our paper from such a distinguished group of researchers. The diversity of approaches represented enriches the practice of our study. To best address these different perspectives, we have organized our responses around each discussion separately. However, there are some common themes that we would like to briefly highlight as we will return to them repeatedly during our rejoinder.

The authors realize the need for clear and detailed descriptions of the data and DPRK population reconstruction procedures so that readers and discussants can easily understand our approaches without any misunderstandings.

\section{Data Sources and Reconstruction of DPRK Population}

\subsection{Population information of DPRK}

Single age-sex specific population and mortality without the military population data were released in the 1993 DPRK census and the 2008 DPRK census released single age-sex specific population with military population data, but the five-year age group and sex military population and mortality were available. One goal of this paper is to estimate excess deaths from the famine in 1995; therefore, we should restore a complete single age-sex specific population with imputed military population in the 1993 census data and a single age-sex specific mortality for the 2008 census data which is required to reconstruct the 1998-2007 population by applying the population component method in a backward direction.

Other official sources for DPRK population are crude death rates of 1996, 1998, and 1999 (Moon, 2011) and crude death rates and life expectancies of 2003, 2004, and 2005 from the reports of Reproductive Health Surveys. These vital statistics were all officially released by the DPRK Central Bureau of Statistics.

\footnotetext{
${ }^{1}$ Corresponding author: Department of Statistics, Korea University, Anam 5-1, Seoul, 136-701, Republic of Korea. Email: yspark@korea.ac.kr

Published 31 January 2015 / journal homepage: http://csam.or.kr (C) 2015 The Korean Statistical Society, and Korean International Statistical Society. All rights reserved.
} 


\subsection{Reconstruction of DPRK population}

The procedures to reconstruct the 1994-2007 population can be summarized in four steps.

\section{Step 1: Restoring the 1993 military population}

The five-year age group and sex specific distribution of the 2008 military population allocate the total 691,029 military population in 1993 into a five-year age group and sex specific military population. We then check if the survival rates exceed (or not) for the five-year age groups of each sex between the 1993 population and the corresponding cohort age group of 2008 . We then adjust the allocated military population so that the survival rates are less than 1 and sufficiently smooth. This smoothing and allocation procedure is also applied to within each of the allocated five-year groups for the 1993 single age-sex specific military population or (equivalently) the complete 1993 single age-sex specific population.

\section{Step 2: Model life tables for DPRK mortality patterns}

First, we fit the Brass-logit model (Brass, 1977) to each of the 1993 and 2008 mortality rates to the Coale-Demeny and UN model life tables. Note that we use five-year age group mortality rates for model fit because the model life tables consist of only five-year age group mortalities with the open ended at 100 years old. The south type model life table shows the best fit to the 1993 mortality versus the east type that best fit the 2008 mortality rates. We then obtain the mortality rates for 1994-1998 from the south type and those for 1999-2007 from the east type, corresponding to respective life expectancies of 1994-2007.

We first estimate single age-sex specific mortality rates of 2008 open ended at 100 years old before proceeding to estimate single age-sex specific mortality rates for 1994-2007 because the 2008 mortality rates are presented by five-year age group mortality of each sex open ended at 80 years old. Note that the 1993 census data provide single age-sex specific mortality rates with the open ended at 100 years old. After estimating the five-years age group mortality rates with the open ended at 100 by applying the Brass-logit model with the five-year age group mortality rates of the east type as the covariate, we estimate single age-sex specific mortality rate with the open-ended at 100 by the Greville smoothing method (Greville, 1945).

\section{Step 3: Mortality Rates for 1994-2007}

We match the life expectancy at birth of the south type with the life expectancies of 1994-1998 (Table 2). This match can be done by linearly interpolating the given life expectancies of model life tables. For example, the mortality rates for males in 1995 with a life expectancy of 67.2 obtained by the south type with a life expectancy of 67 multiplied by 0.8 plus the south type with 68 multiplied by 0.2 . This represents five-year age group mortality rates open ended at 100 years old that are then applied to the Greville smoothing method to produce single age mortality rates. This procedure is repeatedly 
applied for 1994-1998 regardless of sex. The exact same approaches are applied to the east type for 1999-2007 single age-sex specific mortality rates open ended at 100 years old.

Step 4: Reconstructing the population of 1994-2007

We use the population component method under no international migration with the following population balanced equation

$$
P_{t+1}^{a+1}=P_{t}^{a}-D_{t}^{a}=P_{t}^{a}\left(1-d_{t}^{a}\right)
$$

where $P_{t}^{a}, D_{t}^{a}$, and $d_{t}^{a}$ are the population, deaths, and death rate of age $a$ in year $t$, respectively.

Since we have complete single age-sex specific population for the two censuses, 1993 and 2008 , and single age-sex specific mortality rates for all years from 1993 to 2008, we easily estimate the population by applying $P_{t+1}^{a+1}=P_{t}^{a}\left(1-d_{t}^{a}\right)$ forward started at $t=1993$ until $t=1997$ and by $P_{t}^{a}=$ $P_{t+1}^{a+1} /\left(1+d_{t}^{a}\right)$ backward started at $t=2007$ until $t=1998$. Here, for the population at age 0 from 1994 to 1998, the crude birth rates officially released for 1996, 1998, and 1999 (Moon, 2011) are used with the sex ratio at birth constant at 1.05 (Goodkind, 1999). The crude birth rates for 1994, 1995, and 1997 are linearly interpolated. We then have two estimates for the 1998 population, calculated forward from the 1993 census and backward from the 2008 census.

These two estimates showed clear discrepancy in the total population for each sex and reveals a higher 1998 total population from the 1993 census than the 2008 census. This implies that the presumed life expectancies for 1994-1997 (Goodkind and West, 2001) are too high. We revised the life expectancies of 1994-1998 in a way that the total population of each sex (estimated from both of 1993 and 2008 censuses) are consistent based on the assumption that the life expectancies of both 1997 and 1998 are the same (i.e, the same mortality rates). This assumption is partly due to some ambiguity about the onset and end of famine conditions (Goodkind and West, 2001; Lee, 2004; Haggard and Noland, 2007) and partly for convenience of the revision; however, it does not affect estimation of excess deaths from the famine, which is one of our aims in this paper. We further assume that mortality rates in 1995 were twice than other years to accommodate an immediate effect of the onset of the famine, yielding a decline of the life expectancy from 1995 to 1996 that is twice than other years from 1993 to 1998 . The resulting life expectancies are summarized in the second row of Table 2. Using the revised life expectancies which produce consistent total population of each sex in 1998, we a restore single age-sex specific population for 14 years from 1994 to 2007 based on the same method described above.

\section{Responses to Discussants}

Professor Kim, Professor Hwang, and Dr. Gerland addressed their concerns for a unified Korea in various ways along with importance of understanding the DPRK population. As described in our paper, understanding the population structure of DPRK is important to prepare for unification and estimate the socio-economic costs for national welfare under a unified Korea. 
The reunification of divided nations such as Germany can be informative with regards in the changes that may be expected due to reunification; however, the economic gap between South and North Koreas is larger than that of the former East and West Germanys. In the case of German unification, fertility and mortality patterns change according to changes in society (Gjonca et al., 2000). The immediate post-reunification effects in East Germany were a sharp decline in births and marriages accompanied by increased life expectancy and migration consequently, a similar a phenomenon may occur in the unified Korea. Understanding the population structures of both Koreas for the present and future is a salient task; consequently, we conduct a population projection of a unified Korea as a future work based on the reconstructed DPRK population and the projected population of South Korea. This future work will produce various aging indices and dependent ratios under a series of unification time schedules to assess the population aging speed (or degree) and economic potential of a unified Korea.

Professor Keewhan Kim addressed his concerns on the effect of re-unification with respect to economic benefits, population aging, the social security system, and miscellaneous things related to a unified Korea. He also indicated that the optimistic benefit (called a 'bonanza') from reunification can be dangerous without a careful understanding of the DPRK population structure. He encouraged more studies on the DPRK population as long as research is based on logic and clear methodology due to the scarcity of information on the DPRK population. In this regard, he required more precise description for construction of DPRK that is thoroughly addressed by this rejoinder.

Professor Myeongin Hwang provided a precise review of DPRK population projections conducted by Statistics Korea (the Bureau of Census in South Korea). He also provided excellent interpretation on why the number of deaths among women was higher than men and why female seniors were less likely to survive. This nice interpretation definitely enhanced our paper. He additionally raised three questions for future works based on our study. Among them, the one we can do in the near future as statisticians is the third question asking for the population structure of a unified Korea in terms of labor force, international migration, fertility, and aging. A partial answer for this question will appear in our next work.

Dr. Daniel Goodkind provided a thoughtful review of our paper for excess deaths as well as reliability of census data and demographic analysis. In particular, he provided important empirical evidence against the assumption by Spoorenberg and Schwekendiek that life expectancy declined by three years from 2000 to 2008. This empirical evidence (FAO/WFP, 2010) fundamentally strengthens the theoretical reasoning of our paper. We did not recognize that Goodkind et al. (2011) and estimated almost the same number of excess deaths from the famine, ranged between about 500 and 600 thousand; however, their approaches are different from ours in the mortality of the 1993, reconstruction method for the 1994-2007 population, and some minor assumptions. We also agree that the generalized growth balance method by Hill (1987) shows some minor difference in specifications such as selection of age segmentation (Hill et al., 2009; Murray et al., 2010) and underlying data. However, the values of GGB (not near 100\%) indicate the incompleteness of census enumeration and death registration. In this sense, the completeness estimates of Goodkind et al. (2011) and ours indicate the 
same results for the death reporting problems in the two censuses.

Dr. Patrick Gerland (at United Nations Population Division) offered criticism to our paper including issues, questions, problems, and use of English. Although his inconsiderate criticism is painful, it greatly enhances our paper. The main reason why we use the term "inconsiderate" is that most of his criticism seem based on his own insight and limited knowledge toward statistical view in DPRK population analysis.

He criticized the vital statistics used in our paper for life expectancies and crude birth rates between 1993 and 2008 released by North Korea's Central Bureau of Statistics. He asked more than four times for the reliability of these official statistics like: "What is the basis for the official values of life expectancy at birth used by the authors?", "On what empirical data these values were estimated?", and "What assumptions or models were used by official authorities to derive the numbers between 1993 and 2008?". As mentioned in our paper, North Korea had conducted a series of surveys in collaboration with UN and released vital statistics such as life expectancies and crude birth rates through the reports of 2003, 2004, and 2005 Reproductive Health Surveys. Spoorenberg and Schwekendiek (2012) demonstrated that vital statistics such as fertility and mortality are reliable. I think that Dr. Patrick Gerland knows this fact from his question for "on p.9 Table 2" in our paper.

We like to ask him how and why we evaluate these surveys in terms of the questions he raised. Instead, he should give us concrete evidence why the official vital statistics released by North Korea government with help of UN are unreliable. Otherwise, should we believe the many unsubstantiated assumptions that researchers have made for the DPRK population and excess famine related deaths? He seems to have over-viewed that when there is a lack of information or uncertainty about data quality there are multiple ways in which the evidence can be fit together as mentioned in the comments of Dr. Goodkind as a discussant. We also believe that a statistical judgment for a model fit to an empirical data is superior than by simple human observation.

He should misunderstand the available data information of the 1993 and 2008 censuses used to estimate 1993 military population partly because of his limited knowledge and partly because of our incomplete description for the census data and the procedure to estimate the 1993 military population. We now clearly describe them and summarize them in this rejoinder to prevent misunderstanding in our approaches.

Finally, we explain the difference between Spoorenberg and Schwekendiek (2012) and ours, which is raised by Dr. Gerland, by the following three points. First, five-year age group and sex military population in 1993 are constructed similar to Spoorenberg and Schwekendiek. However, the method for the survival rate adjustment is different and we further allocated single age-sex specific military population within the corresponding five-year age group of each sex, keeping the age dependent pattern of survival rate between 1993 and 2008. Spoorenberg and Schwekendiek presented a single age survival between 1993 and 2008 by figure, we do not understand it because the military population data in 2008 are available only for a five-year age group and sex (as described in the early of this rejoinder). Second, Spoorenberg and Schwekendiek assumed three scenarios of life expectancies at birth to esti- 
mate excess deaths and used the observed 2008 mortality pattern as the target mortality pattern under the assumption that the life expectancy of 2000 is the same as 1993 . This is a completely different approach from ours. In particular, Spoorenberg and Schwekendiek's estimates require that life expectancy declined by three years from 2000 to 2008. This is contradicted to the empirical evidence of FAO/WFP (2010) that showed improvement in child health and, most likely, improved health at all ages as indicated in discussion of Goodkind. Third, researchers have proposed different estimates for the number of excess deaths due to some ambiguity about onset and end of famine conditions : Goodkind and West (2001) estimated 600,000 to 1,000,000 excess deaths, Goodkind et al. (2011) estimated 500,000-600,000 and Spoorenberg and Schwekendiek (2012) ranged from 240,000-420,000. Our estimates of excess deaths is 489,972 574,306 which is similar to Goodkind, West and Johnson's estimate.

\section{Acknowledgments}

The authors wish to thank Professor Keewhan Kim for his discussions with the effect of unification and importance of understanding population of North Korea, Professor Myeongin Hwang for his interpretation related to the famine with the reason for the female deaths in a hard time, Dr. Daniel Goodkind for his valuable comments for excess deaths from the famine and the life expectancies with good empirical evidence, and Dr. Patrick Gerland (at United Nations Population Division) for his criticisms and comments which greatly enhanced our paper.

\section{References}

Brass, W. (1977). On the scale of mortality, Biological Aspects of Demography, Taylor \& Francis. Food and Agriculture Organization-World Food Programme (FAO/WFP) (2010). Crop and Food Security Assessment Mission to the Democratic People's Republic of Korea, Special Report, November 16. http://www.fao.org/docrep/013/al968e00.htm

Gjonca, A., Brockmann, H. and Maier, H. (2000). Old-Age Mortality in Germany prior to and after Reunification, Demographic Research, 3.

Goodkind, D. (1999). Do parents prefer sons in North Korea?, Studies in Family Planning, 30, 212 218.

Goodkind, D. and West, L. (2001). The North Korean famine and its demographic impact, Population and Development Review, 27, 219-238.

Goodkind, D., West, L. and Johnson, P. (2011). A reassessment of mortality in North Korea, 19932008. paper presented at the Annual Meeting of the Population Association of America March 31-April 2, Washington, D.C.

Greville, T. N. E. (1945). Actuarial note: Some extensions of Mr. Beers's method of interpolation, American Institute of Actuaries, 34, 21-34.

Haggard, S. and Noland, M. (2007). Famine in North Korea: Markets, Aid, and Reform, Columbia University Press, New York.

Hill, K. (1987). Estimating census and death registration completeness, Asian and Pacific Population Forum, 1, 8-24.

Hill, K., You, D. and Choi, Y. (2009). Death distribution methods for estimating adult mortality: Sensitivity analysis with simulated data error, Demography Research, 21, 235-254. 
Lee, S. (2004). The famine in North Korea, 1994-2000: outbreak, impact, and character, The Korea Institute for National Unification.

Moon, H. E. (2011). Democratic People's Republic of Korea in view of Demography (Japanese), Myungsuk Press.

Murray, C., Rajaratnam, J., Marcus, J., Laakso, T. and Lopez, A. (2010). What can we conclude from death registration? Improved methods for evaluating completeness, PloS Medicine, 7, 1-17.

Spoorenberg, T. and Schwekendiek, D. (2012). Demographic changes in North Korea: 1993-2008, Population and Development Review, 38, 133-158.

Received December 19, 2014; Revised December 27, 2014; Accepted December 29, 2014 